NOT FOR QUOTATION

WITHOUT PERMISSION

OF THE AUTHOR

\title{
INEFFICIENCY OF NASH EQUILIBRIA
}

Pradeep Dubey

July 1983

WP $-83-74$

Working Papers are interim reports on work of the International Institute for Applied Systems Analysis and have received only limited review. Views or opinions expressed herein do not necessarily represent those of the Institute or of its National Member Organizations.

INTERNATIONAL INSTITUTE FOR APPLIED SYSTEMS ANALYSIS A-2361 Laxenburg, Austria 


\section{PREFACE}

The main theme of this paper is that Nash Equilibria of games with smooth payoff functions generally tend to be inefficient (in the Pareto sense).

Andrzej P. Wierzbicki

Chairman

System \& Decision Sciences 


\section{INEFFICIENCY OF NASH EQUILIBRIA}

BY

PRADEEP DUBEY

1. Introduction. The main theme of this paper is that Nash Equilibria

(N.E.) of games with smooth payoff functions are generally Pareto-inefficient. Suppose that a strategic game with $\mathfrak{n}$ players is given by $\mathfrak{n}$ maps

$$
u^{i}: s^{1} \times \ldots \times s^{n} \longrightarrow R \text {, }
$$

$i=1, \ldots, n$, where $s^{i}$ is the strategy-set and $u^{i}$ the payoff function of player $i$. Our result states that if the functions $u^{i}$ are $c^{2}$, then generically (for an open dense set of payoffs): (a) the set of N.E. is finite, (b) if an N.E. is efficient, then at least one player is on a vertex of his strategy-set, (c) if an N.E. is strong, then at most one player is off a vertex of his strategy-set. Note that (b) implies generic inefficiency if the strategy-sets are vertex-free (e.g., manifolds) or if vertices can a priori be ruled out of N.E. in the given case. The result applies to the multi-matrix games of Nash (section 4). Here a vertex corresponds to a pure strategy and, given the special structure of payoff functions, (c) can be strenthened to: if an N.E. is strong every player is using a pure strategy.

That the outcome of noncooperation (N.E.) is generally incompatible with cooperation (efficiency) has been part of the "folklore" of Game Theory as exemplified in the paracigm of the "Prisoner's Dilemma". The purpose of the paper is to put this on a rigorous footing.

This paper is a rewrite of an old version [2]. It is a pleasure to thank J.D. Rogawski for several comments, and in particular for the example in section 3, which is due in its entirety to him. 


\section{The Main Theorem}

Let $N=\{1, \ldots, n\}, n \geqslant 2$, be the set of players, and $s^{i} \subset R^{k(i)}$, $k(i) \geqslant 1$, the strategy-set of player $i$. Here $s^{i}$ is the unit simplex, i.e., $s^{i}=\left\{x \in R_{+}^{k(i)}: \sum_{j=1}^{k(i)} x_{j} \leqslant 1\right\}$. (For more general strategy-sets, see Remark 1.) Fix neighborhoods $v^{i}$ of $s^{i}$ and put $v=v^{1} \times \ldots \times v^{n}$. Let $U$ be the linear space of all $C^{2}$ functions from $V$ to the reals endowed with the $C^{2}$-norm, i.e., for $a 11^{1}$ ) $u$ in $U$, $\|u\|=\sup \left\{\|u(s)\|,\|D u(s)\|,\left\|D^{2} u(s)\right\|: s \in V\right\}$. Our space of noncooperative games will be $(U)^{n}$; for any $u=\left(u^{1}, \ldots, u^{n}\right) \in(U)^{n}, u^{i}$ is the payoff function of player $i$.

For any $s=\left\{s^{i}: i \in N\right\} S=s^{1} \times \ldots \times s^{n}, T \subset N$, and $e=\left\{e^{i}: i \in T\right\} \in X s^{i}$, let $(s \mid e)$ denote the element of $s$ obtained from $s$ by replacing $s^{i}$ by $e^{i}$ for each $i \in T$.

Assume $u=\left(u^{1}, \ldots, u^{n}\right) \in(U)^{n}$ is fixed. A point $s \in S$ is called

(1) T-efficient if there does not exist any point $e \in \underset{i \in T}{X} s^{i}$ such that

$$
\begin{aligned}
& u^{i}(s \mid e) \geqslant u^{i}(s) \text { for all } i \in T \\
& u^{j}(s \mid e)>u^{j}(s) \text { for some } j \in T
\end{aligned}
$$

(2) a Nash Equilibrium (N.E.) if it is T-efficient for all subsets $T$ consisting of one element

(3) efficient if it is T-efficient for $T=N$

(4) a strong Nash Equilibrium if it is T-efficient for all subsets $\mathrm{T} \subset \mathrm{N}$.

1) i.e., it is required that the norm $\|u\|$ of $u$ be finite in order that $u \in U$. (The case when $u$ is required to be defined only on $S$ is more natural, but will follow from the current case--see Remark 2.) 
Let $N(u), E(u), G(u)$ denote the sets of Nash, efficient, strong Nash points of the game $u$.

Theorem There is an open dense set $U_{0}$ of $(U)^{n}$ such that, for $u \in U_{0}$ :

(a) $\mathrm{N}(\mathrm{u})$ is a finite set

(b) if $s=\left(s^{1}, \ldots, s^{n}\right) \in N(u) \cap E(u)$, then at least one $s^{j}$ is a vertex

(c) if $s=\left(s^{1}, \ldots, s^{n}\right) \in G(u)$, then at most one $s^{j}$ is not a vertex.

Proof First we focus on the case when $s^{i}$ is in the interior of $s^{i}(i=1, \ldots, n)$. Let $r(j)=\sum k(i)$. Then $v$ may be viewed as a subset of the Euclidean space ${ }_{R} r(n)$ of dimension $r(n)$. For $s=\left(s^{1}, \ldots, s^{n}\right) \in V, s^{1}$ gives the first $k(1)$ coordinates of $s, s^{2}$ the next $k(2)$ coordinates, i.e., the coordinates from $r(1)+1$ til1 $r(2)$, etc. Consider the derivative map

$$
\text { (U) }{ }^{n} \times V \stackrel{D}{\longrightarrow} R^{n \times r(n)}
$$

given by:

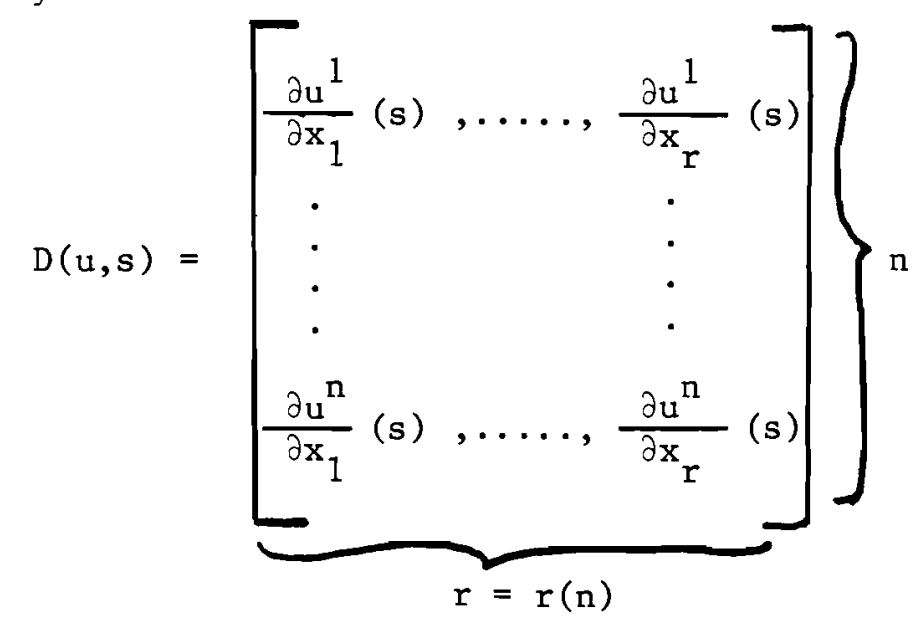

where $u=\left(u^{1}, \ldots, u^{n}\right) \cdot$ For fixed $u$,

$$
\mathrm{V} \stackrel{\mathrm{D}}{\mathrm{u}} \mathrm{R}^{\mathrm{n} \times \mathrm{r}(\mathrm{n})}
$$

is the map obtained by restricting $D$ : 


$$
D_{u}(s)=D(u, s)
$$

We define two subsets of $R^{n \times r(n)}$ that help in the analysis of $N(u)$, $E(u), G(u)$.

$$
N^{*}=\left\{A \in R^{n \times r(n)}: A_{i j}=0 \text { for } r(i-1)+1 \leqslant j \leqslant r(i)\right\}
$$

where $A_{i j}$ denotes the entry in the $i^{\text {th }}$ row and $j^{\text {th }}$ column of matrix $A$, i.e., $A \in N^{*}$ if it has the form

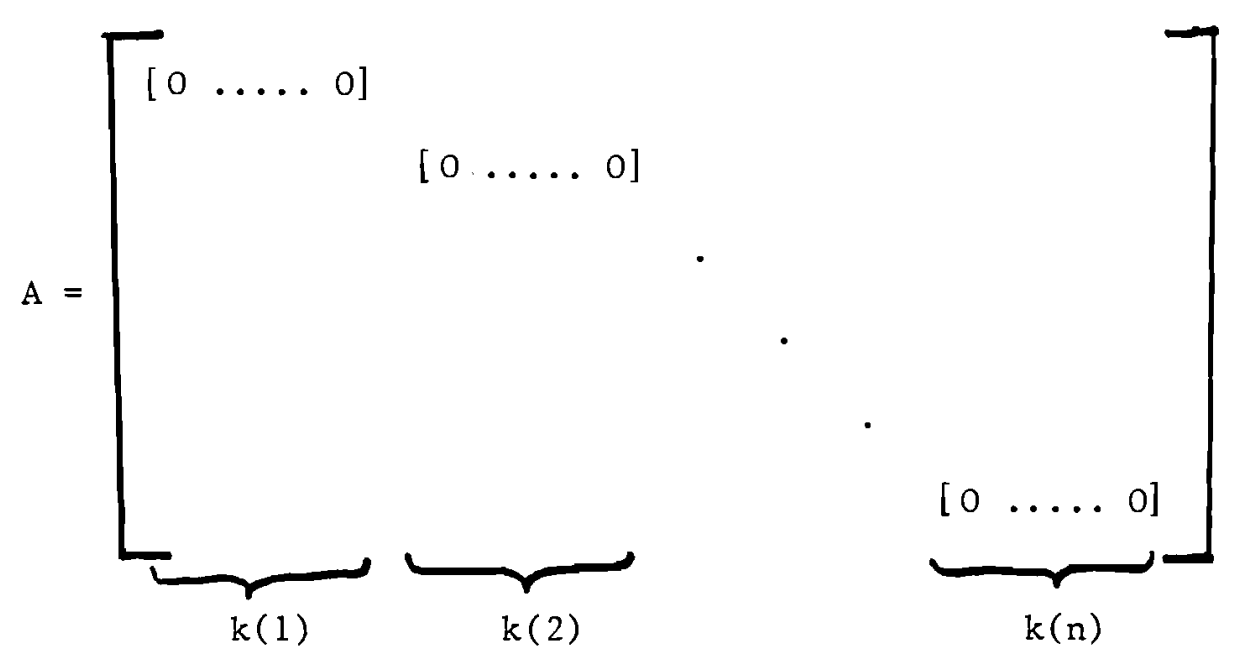

It is clear, since $s \in \operatorname{Int} s^{1} \times \ldots \times$ Int $s^{n}$ (Int $\equiv$ interior), that

$$
s \in N(u) \Rightarrow s \in D_{u}^{-1}\left(N^{*}\right)
$$

Also define $E^{*}=\left\{A \in R^{n \times r(n)}\right.$ : the rows of $A$ are linearly dependent $\}$. Then, as pointed out by Smale in [5] (see Appendix):

$$
s \in E(u) \Rightarrow s \in D_{u}^{-1}\left(E^{*}\right)
$$

From (i) and (ii), we have

$$
\text { (iii) } \quad s \in N(u) \cap E(u) \Rightarrow s \in D_{u}^{-1}\left(N^{*} \cap E^{*}\right)
$$

One can easily check that $N^{*}$ is a submanifold of $\mathrm{R}^{\mathrm{n} \times \mathrm{r}(\mathrm{n})}$ of codimension $r(n)$; and $N^{*} \cap E^{*}$ is a finite union of submanifolds $H_{1}^{*}, \ldots, H_{p}^{*}$ of $R^{n \times r(n)}$ each of which has codimension $\geqslant r(n)+1$ 
Fix compact sets $\underline{v}^{1}, \ldots, \underline{v}^{n}$ such that $s^{i} \subset$ Int $\underline{v}^{i} \subset v^{i}$. Let $M$ be any submanifold of $R^{n \times r(n)}$. We claim

$(*)$ There is an open, dense set $U_{M}$ of $(U)^{n}$ such that, if $u \in U_{M}$, then $D_{u}$ is transverse to $M$ at every $x$ in $\underline{v}=\underline{v}^{1} \times \ldots \times \underline{v}^{n}$.

(*) will follow from the Transveral Density and Openness Theorems (see Appendix) if we can show:

$(* *) \quad D$ is tranverse to $M$ at every $x \in V$.

Let $y$ be an arbitrary vector in $\mathrm{R}^{\mathrm{n} \times \mathrm{r}(\mathrm{n})}$ and consider any $\left(u_{0}, s_{0}\right) \in(U)^{n} x v$. Construct the smooth path $\left.\left(u_{t}, s_{t}\right)\right|_{t=0} ^{1}$ in (U) ${ }^{\mathrm{n}} \mathrm{XV}$ as follows :

$$
\begin{aligned}
& s_{t}=s_{o} \\
& u_{t}^{i}(x)=u_{0}^{i}(x)+\sum_{j=1}^{r=r(n)} t y_{i j} x_{j}
\end{aligned}
$$

Then

$$
D\left(u_{t}, s_{t}\right)=D\left(u_{0}, s_{0}\right)+\left[\begin{array}{ccc}
t_{11} & \ldots . ., & t y_{1 r} \\
\cdot & & \cdot \\
\cdot & & \cdot \\
t y_{n 1}, \ldots ., & t_{n r}
\end{array}\right]
$$

Therefore $\left.\frac{d}{d t} D\left(u_{t}, s_{t}\right)\right|_{t=0}=y, \quad$ verifying 1$)(* *)$, and hence $(*)$. Consider the open dense sets $\mathrm{U}_{\mathrm{N}^{*}}, \mathrm{U}_{\mathrm{H}_{1}^{*}}, \ldots, \mathrm{U}_{\mathrm{H}_{\mathrm{P}}^{*}}$ given by $(*)$ for the

1) That the inverse image of $D$ splits follows trivially from the finite-dimensionality of the range. 
submanifolds $\mathrm{N}^{*}, \mathrm{H}_{1}^{*}, \ldots, \mathrm{H}_{\mathrm{P}}^{*}$. Put $\mathrm{U}^{*}=\mathrm{U}_{\mathrm{N}^{*}} \cap \mathrm{U}_{\mathrm{H}_{1}^{*}} \cap \ldots \cap \mathrm{U}_{\mathrm{H}_{\mathrm{P}}^{*}} \cdot$ Then $U^{*}$ is also open dense in $(U)^{n}$. Moreover it follows from (*) that, for $u \in U^{*}$,

$$
\begin{aligned}
& \operatorname{codim} \mathrm{D}_{\mathrm{u}}^{-1}\left(\mathrm{~N}^{*}\right)=\operatorname{codim} \mathrm{N}^{*}=r(\mathrm{n}) \\
& \operatorname{codim} \mathrm{D}_{\mathrm{u}}^{-1}\left(\mathrm{H}_{\mathrm{j}}^{*}\right)=\operatorname{codim} \mathrm{H}_{\mathrm{j}}^{*}>\mathrm{r}(\mathrm{n})
\end{aligned}
$$

But $\operatorname{dim} V=r(n)$, hence $\operatorname{dim} D_{u}^{-1}\left(N^{*}\right)$ is zero, i.e., $D_{u}^{-1}\left(N^{*}\right)$ is a discrete set. Then $S \cap D_{u}^{-1}\left(N^{*}\right)$ must be finite since $S$ is compact. By (i), $N(u) \subset\left(S \cap D_{u}^{-1}\left(N^{*}\right)\right)$, so $N(u)$ is also finite (for $u \in U^{*}$ ).

Next consider that the set $N(u) \cap E(u)$ for $u \in U^{*}$. By (iii), it is contained in $\bigcup_{j=1}^{p} D_{u}^{-1}\left(H_{j}^{*}\right)$. But $\operatorname{codim} D_{u}^{-1}\left(H_{j}^{*}\right)>r(n)=\operatorname{dim} V$, so the sets $\mathrm{D}_{\mathrm{u}}^{-1}\left(\mathrm{H}_{\mathrm{j}}^{*}\right)$ are empty. This concludes the proof of the theorem for $s \in \operatorname{Int} s^{1} \times \ldots \times$ Int $s^{n}$.

In general, take any $s=\left(s^{1}, \ldots, s^{n}\right) \in S$. Go through the same argument replacing $s^{i}$ by the face of $s^{i}$ which contains $s^{i}$ in its relative interior. If, for some subset $R \subset N$, the $s^{i}$ are vertices for $i \in R$, then look at the subgame among the remaining players in $T=N \backslash R$. The argument shows that for an open dense set of payoffs, if $s$ is an N.E. of the subgame, then it is not T-efficient (and, also, that such N.E. are finite in number). Let $U_{0}$ be the finite intersection of all these open dense sets. 
Remarks

(1) We took strategy-sets to be simplices because they occur for many classes of games (see, e.g., [4], [6] that have traditionally been examined. But, in general, the $S^{i}$ could be a finite union of submanifolds of some Euclidean space (contained in a compact region). The theorem clearly holds. (A vertex would now mean a zero-dimensional piece in this union, when the union is expressed minimally.)

(2) Define a function $u: S \longrightarrow R$ to be $C^{2}$ if it can be extended to a $C^{2}$ function on some neighborhood of $S$. Let $\underline{U}$ be the space of al1 $C^{2}$ functions on $S$ endowed with the norm $\|u\|_{S}=\sup \left\{\|u(s)\|,\|D u(s)\|,\left\|D^{2} u(s)\right\|: s \in s\right\}$

It is more natural to consider $(\underline{U})^{n}$, rather than $(U)^{n}$, to be the space of games. However, our theorem holds for $(\underline{U})^{n}$ also. For any $u \in(U)^{n}$ let $u_{r}$ be the member of $(\underline{U})^{n}$ obtained by restricting $u$ from $V$ to $S$. First note that

(iv) $\quad\|u\|<\varepsilon \Rightarrow\left\|u_{r}\right\|_{S}<\varepsilon$

Also, it is well known that--if the $s^{i}$ are "nice" sets, e.g., simplices, spheres--

(v) There exists a $\mathrm{K}>0$ such that: for any $\underline{\mathrm{u}} \in(\underline{\mathrm{U}})^{\mathrm{n}}$ with $\| \underline{u}_{\mathrm{S}}<\varepsilon$, we can find a $u \in(U)^{\mathrm{n}}$ with $\|\mathrm{u}\|<\mathrm{K} \varepsilon$ and $\mathrm{u}_{\mathrm{r}}=\underline{\mathrm{u}}$. By (iv) and (v), if $U_{0}$ is open dense in $(U)^{n}$, then so is $\underline{\mathrm{U}}_{\mathrm{O}}=\left\{\mathrm{u}_{\mathrm{r}}: \mathrm{u} \in \mathrm{U}_{\mathrm{o}}\right\}$ in $(\underline{\mathrm{U}})^{\mathrm{n}}$.

(3) Let $\tilde{U}$ be any submanifold of (U) ${ }^{n}$ which satisfies the following transversality condition:

(T.C.) The map $\tilde{U} \times V \longrightarrow R^{n \times r(n)}$ is transverse to every submanifold of $R^{n \times r(n)}$

Then, clearly, our theorem holds with $\tilde{U}$ in place of $(U)^{n}$. If 
$\tilde{U}=\tilde{U}^{1} \times \ldots \times \tilde{U}^{n}$ and each $\tilde{U}^{i}$ has the following property:

(T.C.)* for any $y \in R^{r(n)}$ and $x \in V$, there is a smooth path $u_{t}$ in $\tilde{U}^{i}, 0 \leqslant t \leqslant 1$, such that $\frac{d}{d t}\left(D\left(u_{t}, x\right)=y\right.$

then $\tilde{U}$ will satisfy the T.C. condition. The next two sections provide instances of this.

(4) Consider the set $U_{c}$ of $\left(u^{1}, \ldots, u^{n}\right)$ for which each

$$
u_{s}^{i}: v^{i} \longrightarrow R \quad, s \in V
$$

given by $u_{s}^{i}(t)=u^{i}(s \mid t)$ is strictly concave. Then $U_{c}$ is open in $(U)^{n}$. As is well-known $N(u) \neq \emptyset$ if $u \in U_{c}$. This shows that our result is not vacuous.

\section{An Example}

We present the following example because it is particularly simple and illustrates all of the features of the general case.

Consider a game with two players where the strategy-set of each player is the interval $[0,1]$. The payoff functions are then functions on the square $[0,1] \times[0,1]$, which we call $\mathrm{X}$; a point in $\mathrm{X}$ is denoted by $\left(x_{1}, x_{2}\right)$ where $x_{j}$ is the $j^{\text {th }}$ player's strategy choice.

For each point $P=(a, b) \in R^{2}$, let $u_{p}$ be the function

$$
u_{P}(x, y)=-\left[(x-a)^{2}+(y-b)^{2}\right]
$$

i.e., $u_{\mathrm{p}}(\mathrm{x}, \mathrm{y})$ is the negative of the square of the distance from $(\mathrm{x}, \mathrm{y})$ to $P$. Let $U$ be the set $\left\{u_{P}: P \in R^{2}\right\}$ of all such functions. Then $U$ is a submanifold of $C^{2}\left(R^{2}\right)$ and is isomorphic as amanifold to $R^{2}$ itself.

We want to examine that the Nash and efficient sets of the games where each player's payoff function is selected from $U$. A game of this type is determined by assigning a point $P=(\mathrm{a}, \mathrm{b})$ to player 1 and a point 
$Q=(c, d)$ to player 2 , so that their payoff functions are respectively $\mathrm{u}_{\mathrm{P}}$ and $\mathrm{u}_{\mathrm{Q}}$. We denote this game to be $(\mathrm{P}, \mathrm{Q})$.

To find the Nash equilibria of the game $(P, Q)$, note that player l's best response to any strategy choice of player 2 is the point in $[0,1]$ closest to a ; thus his best response is a if a $\varepsilon[0,1], 0$ if $a<0$, and 1 if $a>1$. Similarly player 2's best response to any strategy choice of player 1 is the point in $[0,1]$ closest to $d$.

$$
\text { - } Q=(c, d)
$$

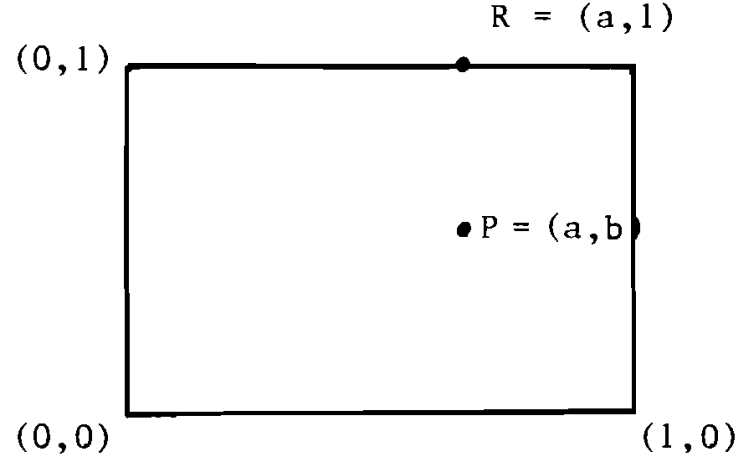

In the figure on the left, the point $R$ is the Nash equilibrium of the game $(P, Q)$. In particular, we see that every game $(P, Q)$ has a unique Nash equilibrium.

To describe the efficient set of the game $(P, Q)$, denoted by $E(P, Q)$, we need a definition. Given any closed convex set $C \subseteq R^{2}$ and a point $M \in R^{2}$, there is a unique closest point to $M$ in $C$. We denote this point by ${ }_{C}(M)$ and call it the retraction of $M$ into $C$. Thus

$$
\text { Distance }\left(M, I_{C}(M)\right)=\underset{P \in C}{\min }(\text { distance }(M, P))
$$

and $r_{C}$ defines a continuous map of $R^{2}$ onto $C$ such that $r_{C}(P)=P$ if $P \in C$.

Lemma 1: For all $P, Q \in R^{2}, E(P, Q)$ is equal to the retraction into $X$ of the line segment joining $P$ and $Q$. In other words, if $L(P, Q)$ is the line segment joining $P$ and $Q$, then $E(P, Q)=r_{X}(L(P, Q))$. Proof: We leave this as a simple exercise for the reader. Note that for all $x \in X$, a point $y$ is a Pareto improvement on $x$ if $y$ lies on the perpendicular drawn from $x$ to the line through $P$ and $Q$. 
Examples: (i) if $P$ and $Q$ both lie in $X$, then $E(P, Q)$ is $L(P, Q)$.

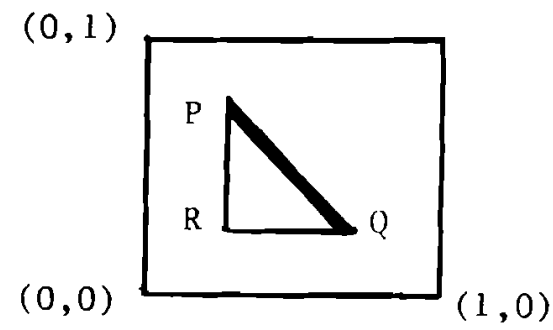

(ii) if $P$ and $Q$ lie outside of $X$, then $E(P, Q)$ may look like the following (the bold line is $E(P, Q)$ ):
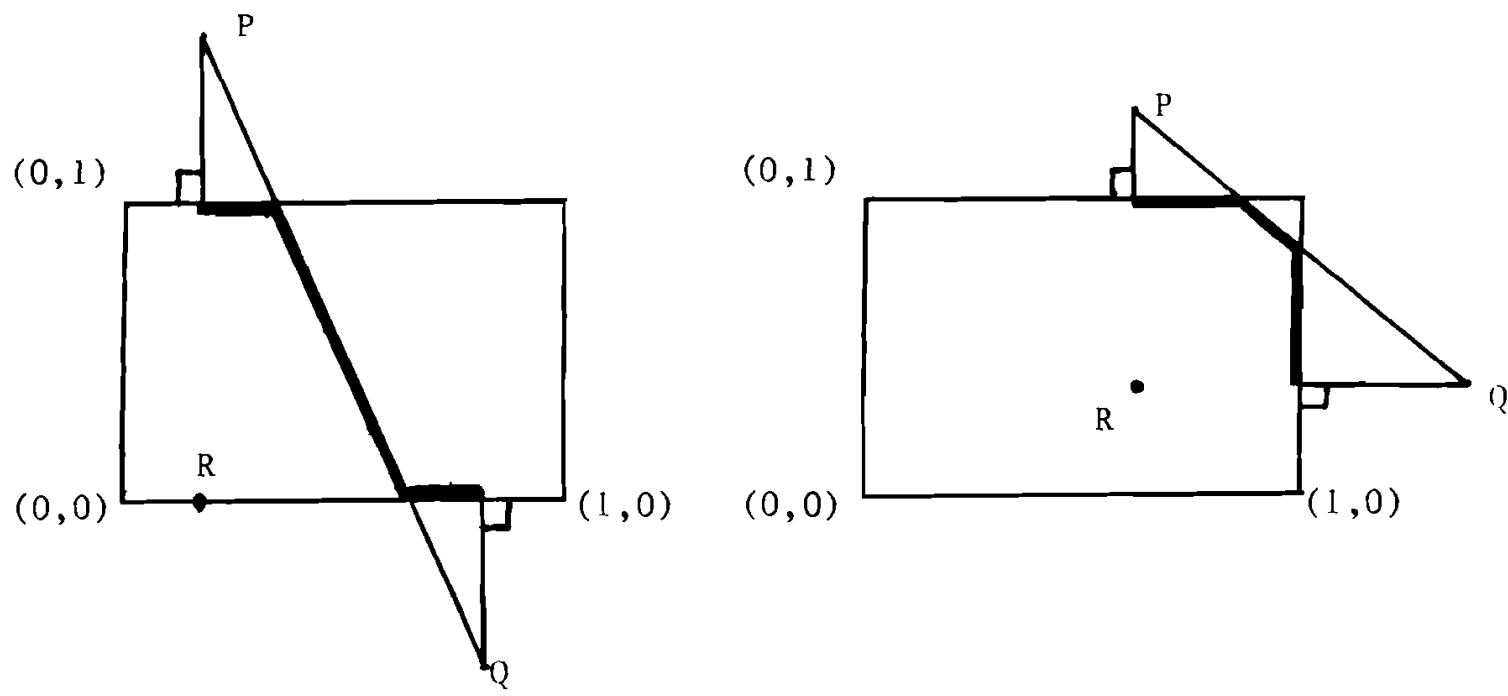

In the examples (i) and (ii), the point $R$ is the unique Nash equilibrium and in both cases it is inefficient.

Lemma 2: Let $R$ be the Nash equilibrium of the game $(P, Q)$ where $P=(a, b)$ and $Q=(c, d)$. Then

(a) If $R$ does not lie on the boundary of $X$ (that is, if neither player is on a vertex of his strategy-set at $R$ ), then $R$ is efficient if and only if $a=c$ and $/$ or $b=d$. The Nash equilibria of nearby games are generically inefficient. 
(b) In the figure below, consider the three games $(P, Q),\left(P^{\prime}, Q^{\prime}\right)$, and ( $\left.P^{\prime \prime}, Q^{\prime \prime}\right)$, with Nash equilibria $R, R^{\prime}$, and $R^{\prime \prime}$ respectively. In these three cases, the Nash equilibrium is efficient and the Nash equilibria of all nearby games remain efficient. In all three cases, at least one player is at a vertex and in the game $\left(P^{\prime}, Q^{\prime}\right)$, the efficient set is reduced to a point.

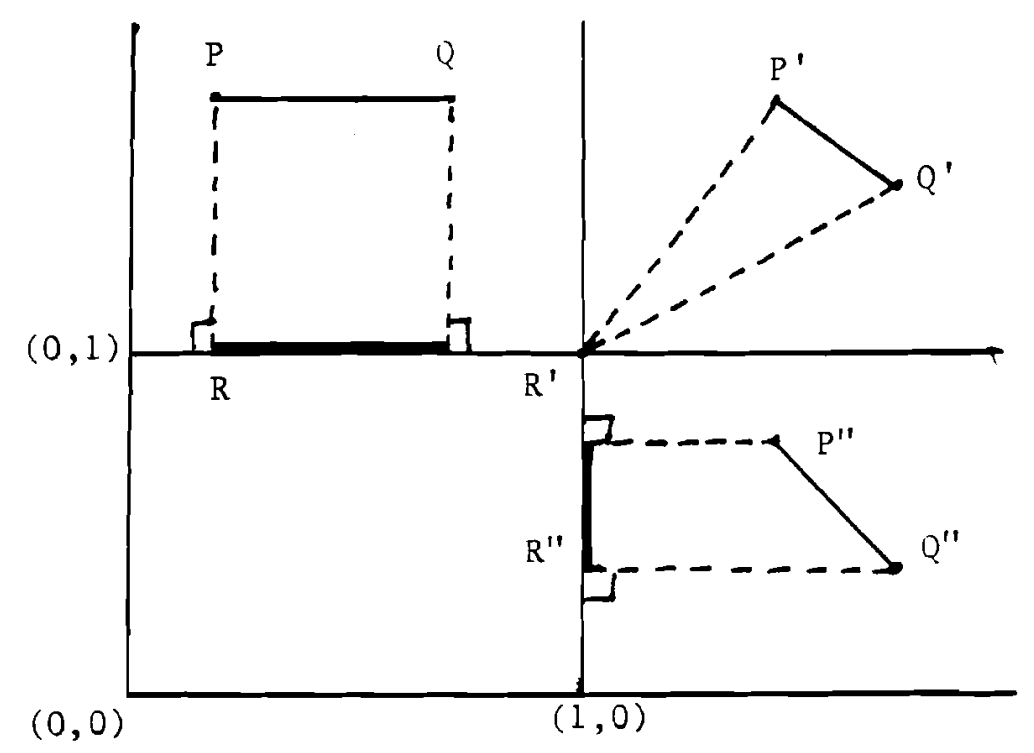

Proof: This is easy to check using Lemma 1.

This example illustrates the following main points of the general theorem:

(i) Nash equilibria are finite in number and vary continuously in $u$.

(ii) Efficient Nash equilibria at which no player is on a vertex of his strategy-set are not robust.

(iii) Robust examples exist of efficient Nash equilibria where at least one player is on a vertex of his strategy-set.

Note that the submanifold of functions $U$ satisfies condition (T.C.)*, as is easily checked, and hence the above results are a consequence of the theorem plus remark ( 3 ). 


\section{Multi-matrix Games}

These were introduced by Nash in [4]. Each player $i$ has a finite set $k_{i}$ of "pure strategies" which we number for convenience as follows:

$$
\begin{aligned}
& \mathrm{K}_{1}=\{1, \ldots, \mathrm{k}(1)\} \\
& \dot{\cdot} \\
& \mathrm{k}_{\mathrm{i}}=\{\mathrm{k}(\mathrm{i}-1)+1, \ldots, \mathrm{k}(\mathrm{i})\} \\
& \dot{\cdot} \\
& \mathrm{K}_{\mathrm{n}}=\{\mathrm{k}(\mathrm{n}-1)+1, \ldots, \mathrm{k}(\mathrm{n})\} .
\end{aligned}
$$

Each $K_{i}$ is now enlarged to a set $x_{i}$ of "mixed strategies", which are simply probability distributions on $\mathrm{K}_{i}$ :

$$
x_{i}=\left\{x \in R^{K_{i}}: \sum_{j \in K_{i}} x_{j}=1, x_{j} \geqslant 0\right\} .
$$

By $R^{K_{i}}$ we mean the Euclidean space of dimension $\operatorname{card}\left(K_{i}\right)$ whose axes are indexed by the elements of $k_{i}$. We identify $k_{i}$ with the set of vertices of $x_{i}$ by associating $j \in K_{i}$ with the point $(0, \ldots, 0, \underbrace{1,}_{j} 0, \ldots, 0) \in R^{K_{i}}$. Let $\mathrm{K}=\mathrm{K}_{1} \times \ldots \times \mathrm{K}_{\mathrm{n}} \cdot$ A multi-matrix game is specified by payoffs: $a^{1} \in R^{K}, \ldots, a^{n} \in R^{K}$. For any $k \in K, a_{k}^{i}$ is the payoff to $i$ if the n-tuple of pure strategies given by $k$ are used. Given $a^{1}, \ldots, a^{n}$ we now define the payoffs $\Pi_{a^{1}}, \ldots, \Pi_{a^{n}}$ on $x=x_{1} \times \ldots \times x_{n}$ as the expectation of the pure strategy payoffs. Let $z_{i}=\left\{x \in R^{K_{i}}: \frac{1}{2}<\sum_{j \in K_{i}} x_{j}\right.$ $\left.<1 \frac{1}{2},\left|x_{j}\right|<2\right\}$ i.e., $z_{i}$ is an open set in $R^{K_{i}}$ which contains the simplex $X_{i}$. Put $Z=z_{1} \times \ldots \times z_{n}$. For $a \in R^{K}$, define $\mathbb{I}_{a}: Z \rightarrow R$ by

$$
\Pi_{a}(x)=\sum_{k \in K} x_{k} a_{k}
$$


where $x_{k}$ denotes $x_{j(1)} \times \ldots \times x_{j(n)}$ for $k=(j(1), \ldots, j(n))$. Then if $a^{i} \in R^{K}$ is the payoff of $i$ in the pure-strategy game, $\Pi_{a}$ restricted to $X$ gives his payoff in its "mixed extension."

To apply the Theorem to this context it will suffice to check that $U=\left\{\Pi_{a}: a \in R^{K}\right\}$ satisfies the (T.C.)* condition for any $z \in Z$. Put $\mathrm{L}=\mathrm{K}_{1} \mathrm{U} \ldots \mathrm{UK_{ \textrm {n } }}=\{1, \ldots, \mathrm{k}(\mathrm{n})\}$. For any $\mathrm{j} \in \mathrm{L}$, let $\mathrm{K}^{-j}=\mathrm{K}_{1} \times \ldots \times \mathrm{K}_{\mathrm{i}-1} \times \mathrm{K}_{\mathrm{i}+1} \times \ldots \times \mathrm{K}_{\mathrm{n}}$ where $\mathrm{i}$ is such that $\mathrm{j} \in \mathrm{K}_{i}$. (Since $L$ is a disjoint union, this is well-defined.) Also for any $q=(\ell(1), \ldots, \ell(i-1), \ell(i+1), \ldots, \ell(n))$ in $k^{-j}$, denote the element $(\ell(1), \ldots, \ell(i-1), j, \ell(i+1), \ldots, \ell(n))$ of $k$ by $(q, j)$. With this notation, we see that $\frac{\partial \Pi_{a}}{\partial x_{j}}(z)=\Sigma_{q \in K^{-j}} z_{q}^{a}(q, j)$ where $z_{q}=z_{\ell(1)} \times \ldots \times z_{\ell(i-1)} \times z_{\ell(i+1)} \times \ldots \times z_{\ell(n)}$ for $q=(\ell(1), \ldots, \ell(i-1), \ell(i+1), \ldots, \ell(n))$. Take any $v \in R^{L}$.

For each $j=1, \ldots, k(n)$ there is clearly a $q(j) \in K^{-j}$ such that $z_{q(j)} \neq 0$. Now consider the path $\left.\Pi_{\mathrm{a}}\right|_{\mathrm{t}=0} ^{1}$ where

$$
a_{k}^{t}= \begin{cases}a_{k}+\left(t v_{j} / z_{q(j)}\right) & \text { if } k=(q(j), j) \\ a_{k} & \text { otherwise }\end{cases}
$$

Then $\frac{d}{d t}\left(\left(D \Pi_{a} t\right)(z)\right)=v$. This shows that $U$ satisfies $(T . C)$.$* at any$ $z \in Z$.

By the Theorem there is an open dense set $V_{1}$ of (IJ) ${ }^{n} \equiv\left(R^{K}\right)^{n}$ such that if $\left(a^{1}, \ldots, a^{n}\right)=a \in v_{1}$ then (a) the N.E. of $\Pi_{a}=\left(\Pi_{a}, \ldots, \pi_{a^{n}}\right)$ are finite in number, (b) if an N.E. of $\Pi_{a}$ is efficient, there is at least 
one player who uses a pure strategy, (r) if an N.E. of $\Pi_{a}$ is strong, then at most one player's strategy is possibly not pure. To sharpen (c), let $V_{2}$ be the subset of (U) ${ }^{n}$ given by

$$
v_{2}=\left\{\left(a^{1}, \ldots, a^{n}\right) \in(U)^{n}: a_{k}^{l} \neq a_{k^{\prime}}^{l^{\prime}} \text { if either } \ell \neq l^{\prime} \text { or } k \neq k^{\prime}\right\}
$$

$\mathrm{V}_{2}$ is open and dense in $(U)^{\mathrm{n}}$. Moreover if $\mathrm{x}=\left(\mathrm{x}_{1}, \ldots, \mathrm{x}_{\mathrm{n}}\right) \in \mathrm{X}$ is an N.E. of $\Pi_{a}$ for $a \in V_{2}$, and if all but one of the players use pure strategies at this N.E., then clearly so does the remainding player. Let $\mathrm{V}=\mathrm{v}_{1} \cap \mathrm{v}_{2}$. We have proved

Theorem (Multi-Matrix): There is an open dense set $V$ of (U) ${ }^{n}$ such that, if $a \in V$,

(a) the N.E. of $\Pi_{a}$ are finite in number*

(b) if an N.E. of $\Pi_{a}$ is efficient, then at least one player uses a pure strategy

(c) if an N.E. of $\Pi_{a}$ is strong, then each player uses a pure strategy.

\section{Generalizations}

Consider a strategy-to-outcome map $\Phi: S \longrightarrow Y$, with player i's utility $u^{i}$ defined on $Y$, and his payoff given by $u^{i}(\Phi(s)),(i=1, \ldots, n)$. (Our theorem focused on the special case when $\Phi$ is the identity map.) If $\operatorname{dim} S=\operatorname{dim} Y$ and $\Phi$ has full rank, then it is clear that our theorem continues to hold by the same proof. However, if $\operatorname{dim} S>\operatorname{dim} Y$ then $N(u)$ will no longer be a finite set for generic $u$, but instead a finite union of submanifolds of positive codimension in $S$. Thus $N(u)$ will not "miss" the efficient set $E(u)$. But it seems probable that (for a generic choice of $u$ and $\Phi)$ the two sets $N(u)$ and $E(u)$ will have

* Finiteness--indeed oddness--of the mixed-strategy N.E. was established by different methods in [3]. 
transversal intersection. Therefore the efficient N.E. will constitute submanifolds of positive codimension within the N.E. manifolds, and the general feature of inefficiency of N.E. will still be maintained. We have not worked out the details of this picture.

\section{APPENDIX}

We recall the results used in this paper.

\section{Part 1 (the quotation is from [1])}

"Let $X$ and $Y$ be $C^{1}$ manifolds, $f: X \rightarrow Y$ a $C^{1}$ map, and $W \subset Y$ a submanifold. We say that $f$ is transversal to $W$ at a point $x \in X$, in symbols: $f \bigcap_{x} w$, iff, where $y=f(x)$, either $y \neq w$ or $y \in W$ and

(1) the inverse image $\left(T_{x} f\right)^{-1}\left(T_{y} W\right)$ splits, and

(2) the image $\left(T_{x} f\right)\left(T_{x} X\right)$ contains a closed complement to $T_{y} W$ in $\mathrm{T}_{\mathrm{y}} \mathrm{Y}$.

We say $f$ is transversal to $W$, in symbols: $f \AA W$, iff $f \AA_{x} W$ for every $x \in X$.

Let $A, X$, and $Y$ be $C^{r}$ manifolds, $C^{r}(X, Y)$ the set of $C^{r}$ maps from $X$ to $Y$, and $\rho: A \rightarrow C^{r}(X, Y)$ a map. For a $\in A$ we write $\rho_{a}$ instead of $\rho(a)$; i.e., $\rho_{a}: X \rightarrow Y$ is a $C^{r}$ map. We say $\rho$ is a $C^{r}$ representation iff the evaluation map

$$
\mathrm{ev}_{\rho}: \mathrm{A} \times \mathrm{X} \rightarrow \mathrm{Y}
$$

given by

$$
\mathrm{ev}_{\rho}(\mathrm{a}, \mathrm{x})=\rho_{\mathrm{a}}(\mathrm{x})
$$

for $a \in A$ and $x \in X$ is $a C^{r}$ map from $A \times X$ to $Y$. 
Transversal Density Theorem. Let A, X, Y be $C^{r}$ manifolds, $\rho: A \rightarrow C^{r}(X, Y)$ a $C^{r}$ representation, $W \subset Y$ a submanifold (not necessarily closed), and $\mathrm{ev}_{\rho}: A \times X \rightarrow Y$ the evaluation map. Define $A_{W} \subset A$ by

$$
\mathrm{A}_{\mathrm{W}}=\left\{\mathrm{a} \in \mathrm{A} \mid \rho_{\mathrm{a}} \boldsymbol{K} \mathrm{W}\right\}
$$

Assume that:

(1) $\mathrm{X}$ has finite dimension $\mathrm{n}$ and $\mathrm{W}$ has finite codimension

$$
\mathrm{q} \text { in } \mathrm{Y} \text {; }
$$

(2) $A$ and $X$ are second countable;

(3) $r>\max (0, n-q)$;

(4) $\operatorname{ev}_{\rho} \hbar \mathrm{w}$.

Then $A_{W}$ is residual (and hence dense) in $A$.

Openness of Transversal Intersection. Let $A, X$, and $Y$ be $C^{1}$ manifolds with $X$ finite dimensional, $W \subset Y$ a closed $C^{1}$ submanifold, $\mathrm{K} \subset \mathrm{X}$ a compact subset of $\mathrm{X}$, and $\rho: A \rightarrow C^{1}(X, Y)$ a $C^{1}$ pseudorepresentation. Then the subset $A_{K W} \subset A$ defined by

$$
\mathrm{A}_{\mathrm{KW}}=\left\{a \in \mathrm{A} \mid \rho_{\mathrm{a}} \bar{\aleph}_{\mathrm{x}} \mathrm{W} \text { for } \mathrm{x} \in \mathrm{K}\right\}
$$

is open. This holds even if $\mathrm{X}$ is not finite dimensional, provided that $\rho$ is a $C^{1}$ representation."

For our purposes, it is enough to note that every $C^{1}$ representation is a $C^{1}$ pseudorepresentation. Also $T_{y} W$ is the tangent space to $W$ at $y ; T_{x} f: T_{x} X T_{y} Y$ is the derivative map of $f$ at $x$. See [1] for detailed definitions. 
Part 2 (the quotation, with minor modifications, is from [6]

"Let $u^{i}: W \rightarrow R$ be smooth (i.e., $C^{r}, r \geqslant 1$ ) functions

$(i=1, \ldots, n)$ where $W$ is a manifold in some finite dimensional

Euclidean space. Assume that $\operatorname{dim} w \geqslant n$ throughout. Consider $u=\left(u^{1}, \ldots, u^{n}\right)$, $u: W \rightarrow R^{n}$. At any $x \in W$, the derivative of $u$ at $x, D u(x)$, is a

Iinear map from $T_{x} W$ to $R^{n}$ (made up of $D^{i}(x): T_{x} W \rightarrow R, i=1, \ldots, n$ ).

Proposition. Given $\mathrm{W}$ and $\mathrm{u}$ as above, $\mathrm{x} \in \mathrm{W}$ is an efficient point of

$\mathrm{u}$ iff $\exists \lambda_{i} \geqslant 0, i=1, \ldots, \mathrm{n}$, not all zero with

$\sum_{i=1}^{n} \lambda_{i} D u_{i}(x)=0$ for all $x \in T_{x} W . "$

\section{REFERENCES}

[1] Abraham, R., and J. Robbin, 1976. Transversal Mappings and Flows

(W. A. Benjamin, New York).

[2] Dubey, P., 1978. "Finiteness and Inefficiency of Nash Equilibria", Cowles Foundation Discussion Paper 508R.

[3] Harsanyi, J., 1973. "Oddness of the Number of Equilibrium Points: A New Proof," International Journal of Game Theory, Vol. 2, Issue 4, PP. 235-250.

[4] Nash, J. F., 1951. "Noncooperative Games," Annals of Mathematics, 54, PP. 128-140.

[5] Smale, S., 1973. "Optimizing Several Functions," Proceedings of the Tokyo Manifolds Conference.

[6] Shapley, L. S., and M. Shubik, 1977. "Trade Using One Commodity as a Means of Payment," Journal of Political Economy, 85, pP. 937-968. 